\title{
The 3-ranks of tame kernels of cubic cyclic number fields
}

\author{
by \\ Xuejun Guo (Nanjing)
}

1. Introduction. The purpose of this paper is to generalize the following theorem proved by Jerzy Browkin to general cubic cyclic number fields.

Theorem 1.1 ([1, Theorem 2.4(iii)]). Let $F$ be a cubic cyclic number field with only one ramified prime $p>7$. Then $3 \mid \# K_{2} \mathcal{O}_{F}$ if and only if $p \equiv 1(\bmod 18)$.

Browkin gave two proofs in [1]. The first proof is analytic and depends on deep results by Mazur and Wiles, while the second one is algebraic, using an exact sequence in $K$-theory. In this paper, combining the same exact sequence and Gerth's theory of the 3-class groups of cubic cyclic number fields, we can deal with cubic cyclic number fields with arbitrarily many ramified primes. The main theorem of this paper is Theorem 4.4. From this theorem, one can get the 3-rank formula for general cubic cyclic number fields. As an application, we prove the following theorem in Section 4.

TheOREM 1.2. Let $F$ be a cubic cyclic number field with only two ramified primes $p_{1}, p_{2}$, where $p_{1}<p_{2}$. Then

(1) $2 \leq 3$-rank $K_{2} \mathcal{O}_{F} \leq 4$ if $p_{i} \equiv 1(\bmod 9)$ for $i=1,2$;

(2) 3 -rank $K_{2} \mathcal{O}_{F}=0$ if $p_{1}=3$ and $p_{2} \equiv 4$ or $7(\bmod 9)$;

(3) $1 \leq 3$-rank $K_{2} \mathcal{O}_{F} \leq 3$ otherwise.

2. The 3-rank of the class group of a cubic cyclic number field $F$. Let $F$ be a cubic cyclic number field. Let $p_{1}, \ldots, p_{s}$ be all rational primes which are ramified in $F$ and different from 3 . Then $p_{i} \equiv 1(\bmod 6)$ for $1 \leq i \leq s$. Hence $p_{i}$ is split in $E=\mathbb{Q}(\zeta)$, where $\zeta$ is a fixed primitive cube root of unity. Suppose $p_{i}=\pi_{i} \bar{\pi}_{i}$, where $\bar{\pi}_{i}$ is the conjugate of $\pi_{i}$. We can

2000 Mathematics Subject Classification: 11R70, 19F15.

Key words and phrases: tame kernels, class groups.

Supported by NSFC 10401014, and partially supported by the Natural Science Foundation of Jiangsu province of China (BK 2005207). 
further assume that $\pi_{i} \equiv \bar{\pi}_{i}(\bmod 3)$, otherwise we replace $\pi_{i}$ by $\zeta \pi_{i}$ or $\zeta^{2} \pi_{i}$. Let $K=E F$. By Theorem 2 of [10] and Theorem 2 of [11], $K=E(\sqrt[3]{\beta})$ with

$$
\beta=\frac{\pi_{1} \cdots \pi_{s}}{\bar{\pi}_{1} \cdots \bar{\pi}_{s}} \zeta^{j},
$$

where $j=0,1,2$; and $j=0$ if and only if 3 is not ramified in $E$. Let $t=s$ if 3 is not ramified in $F$, and $t=s+1$ if 3 is ramified in $F$. Let $\mathfrak{P}_{i}$ be the prime in $F$ above $p_{i}, i=1, \ldots, t$. If $t=s+1$, then $\mathfrak{P}_{t}$ is the prime above 3 .

Let $\beta_{i}=\pi_{i} / \bar{\pi}_{i}, i=1, \ldots, s ; \beta_{s+1}=\zeta$ if 3 is ramified in $F$. Let $F_{i}$ be the maximal real subfield of $E\left(\sqrt[3]{\beta_{i}}\right)$. Then $F_{i}$ is a cubic cyclic extension of $\mathbb{Q}$. Let $M=F_{1} \cdots F_{t}$. Recall that the genus field of $F$ is the maximal absolute abelian number field containing $F$, which is unramified at all the finite prime ideals of $F$ (see page 3 of Chapter 1 of [7] for details). It is easy to see that $M$ is an absolute abelian number field with the degree $[M: F]=3^{t-1}$ and $M / F$ is unramified. By Example 10 in Chapter 6 of [7] or Theorem 2.16 of [2], $M$ is the genus field of $F$. The Galois group $\operatorname{Gal}(M / F)$ is an elementary 3 -group of rank $t-1$.

Let $X$ be the group of characters of $\operatorname{Gal}(M / F)$. Let $S_{F}$ be the 3-class group of $F$. Then any element in $X$ is also a character of $S_{F}$ via the Artin maps.

Let $\chi_{1}, \ldots, \chi_{t-1}$ be a basis of $X$, and $A=\left(a_{i j}\right)_{(t-1) \times t}$ be a matrix, where $a_{i j} \in \mathbb{F}_{3}=$ finite field of 3 elements and

$$
\zeta^{a_{i j}}=\chi_{i}\left(\mathfrak{P}_{j}\right), \quad 1 \leq i \leq t-1,1 \leq j \leq t .
$$

Let $r$ be the rank of $A$.

Theorem 2.1 ([12, Theorem]). The 3 -rank of $S_{F}$ is $2 t-2-r$.

Let $\tau$ be a generator of $\operatorname{Gal}(F / \mathbb{Q})$. Let $C_{0}$ be the set of 3-torsion elements in $S_{F}$. Let $\Delta=1-\tau$, ker $\Delta$ the kernel of

$$
\Delta: C_{0} \rightarrow C_{0}, \quad x \mapsto x / \tau(x),
$$

and $\Delta C_{0}$ the image of $\Delta$. Then by the proof of the above theorem in [12], one can see that the 3 -rank of ker $\Delta$ is $t-1$ and the 3 -rank of $\Delta C_{0}$ is $t-1-r$.

3. The 3-rank of the class group of the sextic cyclic number field $K$. In last section, we chose $\tau$ as a generator of $\operatorname{Gal}(F / \mathbb{Q})$. Since $\operatorname{Gal}(K / E) \simeq \operatorname{Gal}(F / \mathbb{Q})$, this $\tau$ can be extended to a generator of $\operatorname{Gal}(K / E)$. Let $S_{K}$ be the 3 -class group of $K, S_{K}^{(\tau)}=\operatorname{ker}\left(\Delta: S_{K} \rightarrow S_{K}\right)$ the group of ambiguous ideal classes, $S_{K}^{1-\tau}$ the image of $\Delta$.

Recall that $K=E(\sqrt[3]{\beta})$, where $\beta=\pi_{1} \bar{\pi}_{1}^{-1} \cdots \pi_{s} \bar{\pi}_{s}^{-1} \zeta^{j}$. Let $d$ be the number of primes that ramify in $K / E$. Then

$$
d= \begin{cases}2 s=2 t & \text { if } 3 \text { is not ramified in } F, \\ 2 s+1=2 t-1 & \text { if } 3 \text { is ramified in } F .\end{cases}
$$


Let $t_{K}$ be the 3-rank of $S_{K}^{(\tau)}$. By Proposition 5.1 of [3],

$$
t_{K}= \begin{cases}d-1 & \text { if each } \pi_{i} \equiv 1\left(\bmod \lambda^{3}\right), \\ d-2 & \text { if some } \pi_{i} \equiv 4 \operatorname{or} 7\left(\bmod \lambda^{3}\right),\end{cases}
$$

where $\lambda=1-\zeta$.

Theorem 3.1 ([3, Theorem 3.1]). The 3-rank of $S_{K}$ is $2 t_{K}-s_{K}$, where $t_{K}$ is the 3 -rank of $S_{K}^{(\tau)}$ and $s_{K}$ is the 3 -rank of $\left(S_{K}^{(\tau)} S_{K}^{1-\tau}\right) / S_{K}^{1-\tau}$.

By (3.1), (3.2) and Theorem 3.1, we have

$$
\text { 3-rank } S_{K}= \begin{cases}4 t-s_{K}-2 & \text { if each } \pi_{i} \equiv 1\left(\bmod \lambda^{3}\right) \text { and } \\ & 3 \text { is not ramified in } F \\ 4 t-s_{K}-6 & \text { if some } \pi_{i} \equiv 4 \text { or } 7\left(\bmod \lambda^{3}\right) \text { and } \\ & 3 \text { is ramified in } F \\ 4 t-s_{K}-4 & \text { otherwise. }\end{cases}
$$

4. The 3-rank of the tame kernel of $F$. Recall that $C_{0}=\left\{x \in S_{F} \mid\right.$ $\left.x^{3}=1\right\}$. Let $C_{0}^{\prime}=\left\{x \in S_{K} \mid x^{3}=1\right\}$. Then it is easy to see that $C_{0}$ is a subgroup of $C_{0}^{\prime}$. Let $\sigma$ be the nontrivial element in $\operatorname{Gal}(K / F)$. We will prove that $C_{0}=C_{0}^{\prime+}=\left\{x \in C_{0}^{\prime} \mid x^{\sigma}=x\right\}$.

For any $x \in C_{0}^{\prime+}$, there is an unramified prime $\mathfrak{P}$ such that the ideal class $[\mathfrak{P}]=x$ by the Chebotarev density theorem. If $\mathfrak{P}$ is inert, then $x \in C_{0}$. If $\mathfrak{P}$ is split, then $x x^{\sigma} \in C_{0}$. Since $x=x^{\sigma}, x^{2} \in C_{0}$. Hence $x^{-1}=x^{2} \in C_{0}$, which implies $x \in C_{0}$. So $C_{0}=C_{0}^{\prime+}$. Let $C_{0}^{\prime-}=\left\{x \in C_{0}^{\prime} \mid x^{\sigma}=x^{-1}\right\}$.

Lemma 4.1. The 3-rank of $C_{0}^{\prime-}$ is equal to 3 -rank $S_{K}-3$-rank $S_{F}$.

Proof. By Lemma 2.1 of [4], $C_{0}^{\prime}=C_{0}^{\prime+} \times C_{0}^{\prime-}$. Since 3-rank $S_{F}=3$-rank $C_{0}$ $=3$-rank $C_{0}^{\prime+}$ and 3-rank $S_{K}=3$-rank $C_{0}^{\prime}$, we can see the 3 -rank of $C_{0}^{\prime-}$ is equal to 3-rank $S_{K}-3$-rank $S_{F}$.

Let $\mathfrak{P}$ be a 3 -adic prime of $K$. Then $\mathfrak{P}$ is ramified in $K / F$. So $\mathfrak{P}$ is fixed by the nontrivial element $\sigma \in \operatorname{Gal}(K / F)$. Let $S_{K, 3}$ be the 3-class group of $\mathcal{O}_{K}[1 / 3]$, and $S_{K, 3}^{-}=\left\{x \in S_{K, 3} \mid x^{\sigma}=x^{-1}\right\}$.

Lemma 4.2. The 3 -rank of $S_{K, 3}^{-}$is equal to the 3 -rank of $C_{0}^{\prime-}$.

Proof. Since the 3-adic primes of $K$ are fixed by $\sigma, S_{K, 3}^{-}=S_{K}^{-}$by Lemma 2.1 of [4]. Hence the 3 -rank of $S_{K, 3}^{-}$is equal to the 3-rank of $S_{K}^{-}$, which is equal to the 3 -rank of $C_{0}^{\prime-}$.

The following is Theorem 3.3 of [13]. Here we give a different proof.

Theorem 4.3 ([13, Theorem 3.3]).

$$
3 \text {-rank } K_{2} \mathcal{O}_{F}=3 \text {-rank } S_{K}-3 \text {-rank } S_{F} \text {. }
$$


Proof. Let $\mu_{3}=\left\{1, \zeta, \zeta^{2}\right\}$. Then by Theorem 5.4 of [8], we have the exact sequence

$$
1 \rightarrow\left(\mu_{3} \otimes S_{K, 3}\right)^{\operatorname{Gal}(K / F)} \rightarrow K_{2} \mathcal{O}_{F} / 3 \rightarrow \bigoplus_{\wp \in S^{\prime}} \mu_{3} \rightarrow 1,
$$

where $S^{\prime}$ is the set of 3 -adic primes of $F$ which split completely in $K$. Since all the 3-adic primes of $F$ are ramified in $K$, it follows that $S^{\prime}$ is empty. For any $x \otimes I \in \mu_{3} \otimes S_{K, 3},(x \otimes I)^{\sigma}=x^{-1} \otimes I^{\sigma}=x \otimes\left(I^{-1}\right)^{\sigma}$. Hence the 3-rank of $\left(\mu_{3} \otimes S_{K, 3}\right)^{\operatorname{Gal}(K / F)}$ is equal to that of $S_{K, 3}^{-}$. By Lemma 4.2, 3-rank $K_{2} \mathcal{O}_{F}=3$-rank $S_{K}-3$-rank $S_{F}$.

THEOREM 4.4. With notations as above, we have

$$
\text { 3-rank } K_{2} \mathcal{O}_{F}= \begin{cases}2 t-s_{K}+r & \text { if each } \pi_{i} \equiv 1\left(\bmod \lambda^{3}\right) \text { and } \\ & 3 \text { is not ramified in } F, \\ 2 t-s_{K}+r-4 & \text { if some } \pi_{i} \equiv 4 \text { or } 7\left(\bmod \lambda^{3}\right) \text { and } \\ & 3 \text { is ramified in } F, \\ 2 t-s_{K}+r-2 & \text { otherwise. }\end{cases}
$$

Proof. This follows from Theorem 2.1, Theorem 4.3 and (3.3).

The first part of the next theorem is Theorem 2.4 of [1], and the second part is Theorem 3.6 of [13]. Here we will give a different proof.

TheOrem 4.5 (J. Browkin, H. Y. Zhou). If $F$ is a cubic cyclic number field with only one ramified prime $p$, then $3 \mid \# K_{2} \mathcal{O}_{F}$ if and only if $p \equiv 1$ $(\bmod 18)$. And if $p \equiv 1(\bmod 18)$, then $3-\operatorname{rank} K_{2} \mathcal{O}_{F} \leq 2$.

Proof. If $p>7$, the first part of this theorem is Theorem 1.1 in the introduction. For $p=3$ or 7 , i.e., $F=\mathbb{Q}\left(\zeta_{7}+\zeta_{7}^{-1}\right)$ or $\mathbb{Q}\left(\zeta_{9}+\zeta_{9}^{-1}\right)$, see [6], [9], or [5].

By Theorem 2.1, we have $t=1$ and $r=0$. Hence 3-rank $K_{2} \mathcal{O}_{F}>0$ if and only if we are in the first case of (4.1). It is easy to see that for a prime $\pi \in \mathcal{O}_{E}, \pi \equiv 1\left(\bmod \lambda^{3}\right)$ if and only if $p=\pi \bar{\pi} \equiv 1(\bmod 9)$. We have already seen that $p$ is an odd prime. So $p \equiv 1(\bmod 18)$, which implies $3 \mid \# K_{2} \mathcal{O}_{F}$ if and only if $p \equiv 1(\bmod 18)$. And if $p \equiv 1(\bmod 18)$, then $t_{K}=1$ by $(3.2)$. Hence 3 -rank $K_{2} \mathcal{O}_{F} \leq 2$.

Recall that the genus field of $K / E$ is the maximal abelian extension of $E$ contained in the Hilbert class field of $K$.

LEMma 4.6. Let $\zeta_{9}$ be a primitive 9th root of unity, and $p$ a rational prime satisfying $p \equiv 4$ or $7(\bmod 9)$. Let $F$ be a cubic cyclic number field which ramifies only at 3 and $p$. Then 
(1) $M=F\left(\zeta_{9}\right)$ is the genus field of $K / E$.

(2) $S_{F}=S_{K} \simeq \mathbb{Z} / 3 \mathbb{Z}$.

Proof. (1) First, we will prove that $M / K$ is unramified. It is easy to see that only 3 may be ramified in $M / K$. Let $\zeta_{p}$ be a primitive $p$ th root of unity, and $F^{\prime}$ the unique cubic subfield of $\mathbb{Q}\left(\zeta_{p}\right)$. Then by Theorem 2 of [10] and Theorem 2 of [11],

$$
F^{\prime}(\zeta)=\mathbb{Q}(\zeta, \sqrt[3]{\pi / \bar{\pi}})
$$

where $\pi \in E=\mathbb{Q}(\zeta)$ is a prime above $p$ and $\pi \equiv \bar{\pi}(\bmod 3)$. By the same theorems,

$$
F(\zeta)=\mathbb{Q}\left(\sqrt[3]{\zeta^{j} \pi / \bar{\pi}}\right)
$$

where $j=1$ or 2 . Hence we have

$$
M=F\left(\zeta_{9}\right)=\mathbb{Q}\left(\zeta_{9}, \sqrt[3]{\pi / \bar{\pi}}\right)=F^{\prime}\left(\zeta_{9}\right) \subset \mathbb{Q}\left(\zeta_{9 p}\right) .
$$

Since the ramification index of 3 in $\mathbb{Q}\left(\zeta_{9 p}\right) / \mathbb{Q}$ is 6 , the ramification index of 3 in $M / F$ cannot exceed 6 . Hence 3 must be unramified in $M / F$, for the ramification index of 3 in $K / \mathbb{Q}$ is 6 .

By $(3.2), t_{K}=1$, which implies the genus field should be an extension of $K$ with degree 3 . We have already seen that $[M: K]=3$ and $M / K$ is abelian unramified. So $M$ is the genus field of $K / E$.

(2) Let $\mathfrak{P}_{2}$ be an ideal of $K$ above $p$. Since $p \equiv 4$ or $7(\bmod 9), \mathfrak{P}_{2}$ is inert in $K\left(\zeta_{9}\right) / K$. The Artin symbol

$$
\left(\frac{M / K}{\mathfrak{P}_{2}}\right)
$$

is not trivial. Since $M / K$ is an abelian unramified extension, the Artin map

$$
\left(\frac{M / K}{\bullet}\right): S_{K} \rightarrow \operatorname{Gal}(M / K)
$$

is surjective. Hence $\mathfrak{P}_{2}$ is not principal. So $s_{K}=1$ by Theorem 4.1 of [3]. Hence 3 -rank $S_{K}=1$. Since 3 -rank $S_{F} \geq 1$ and 3-rank $S_{K} \geq 3$-rank $S_{F}$, we have

$$
S_{F}=S_{K} \simeq \mathbb{Z} / 3 \mathbb{Z}
$$

THEOREM 4.7. Let $F$ be a cubic cyclic number field with only two ramified primes $p_{1}, p_{2}$, where $p_{1}<p_{2}$. Then

(1) $2 \leq 3$-rank $K_{2} \mathcal{O}_{F} \leq 4$ if $p_{i} \equiv 1(\bmod 9)$ for $i=1,2$;

(2) 3 -rank $K_{2} \mathcal{O}_{F}=0$ if $p_{1}=3$ and $p_{2} \equiv 4$ or $7(\bmod 9)$;

(3) $1 \leq 3$-rank $K_{2} \mathcal{O}_{F} \leq 3$ otherwise.

Proof. (1) If $p_{i} \equiv 1(\bmod 9), i=1,2$, then $t=2, t_{K}=3, s_{K} \leq t_{K}$. By the discussion following Theorem 2.1, $r \leq 1$. By the first case of (4.1) in 
Theorem 4.4,

$$
5 \geq 3 \text {-rank } K_{2} \mathcal{O}_{F}=2 t-s_{K}+r \geq 1+r \geq 1,
$$

and 3-rank $K_{2} \mathcal{O}_{F}=1$ if and only if $s_{K}=3$ and $r=0$.

If $s_{K}=3$, then by Corollary 3.2 of [3],

$$
S_{K}=S_{K}^{(\tau)} \simeq \mathbb{Z} / 3 \mathbb{Z}
$$

Hence $S_{K}^{1-\tau}$ is trivial. However, $r=0$ if and only if $S_{F}^{1-\tau} \simeq \mathbb{Z} / 3 \mathbb{Z}$, which contradicts the triviality of $S_{K}^{1-\tau}$. So 3-rank $K_{2} \mathcal{O}_{F} \geq 2$.

Let $\mathfrak{P}_{1}, \mathfrak{P}_{2}$ be two primes in $K$ such that $\mathfrak{P}_{i} \mid p_{i}, i=1,2$; and $\wp_{1}, \wp_{2}$ two primes in $F$ such that $\wp_{i} \mid p_{i}, i=1,2$. Recall that $r$ is the rank of the matrix $A$ defined in Section 2. Let $M$ be the genus field of $K / E$, and $N$ the genus field of $F$. Then $[M: K]=3^{3}$ and $[N: F]=3$. Let $\chi$ be the generator of the group of characters of $\operatorname{Gal}(N / F)$. Via the Artin maps, $\chi$ is also a character of $S_{F}$. Then

$$
A=\left(\chi\left(\wp_{1}\right) \chi\left(\wp_{2}\right)\right) .
$$

Let $N^{\prime}=N(\zeta)$. Then $N^{\prime} \subset M$. If $r=1$, without loss of generality we can assume that $\chi\left(\wp_{1}\right) \neq 1$. So $\wp_{1}$ is not principal, which implies that $\mathfrak{P}_{1}$ is not principal. Let

$$
x_{1}=\pi_{1} / \bar{\pi}_{1},
$$

where $\pi_{1} \in E$ such that $\pi_{1} \bar{\pi}_{1}=p_{1}$ and $\pi_{1} \equiv \bar{\pi}_{1}(\bmod 3)$. Then $N^{\prime}=$ $K\left(\sqrt[3]{x_{1}}\right)$. By Theorem 4.1 of [3], there are $x_{2}, x_{3} \in K$ such that $M=$ $K\left(\sqrt[3]{x_{1}}, \sqrt[3]{x_{2}}, \sqrt[3]{x_{3}}\right)$. Since $\chi\left(\wp_{1}\right) \neq 1, \wp_{1}$ is inert in $N$. Hence $\mathfrak{P}_{1}$ is inert in $N^{\prime}$, which implies that the Artin symbol

$$
\left(\frac{K\left(\sqrt[3]{x_{1}}\right) / K}{\mathfrak{P}_{1}}\right)
$$

is not trivial. So $s_{K} \geq 1$.

By (4.2), 3-rank $K_{2} \mathcal{O}_{F}=5$ if and only if $s_{K}=0$ and $r=1$, which is impossible by the last paragraph. Hence 3 -rank $K_{2} \mathcal{O}_{F} \leq 4$.

(2) If $p_{1}=3$ and $p_{2} \equiv 4$ or $7(\bmod 9)$, then $t=2, t_{K}=s_{K}=r=1$ by Lemma 4.6. Hence 3 -rank $K_{2} \mathcal{O}_{F}=0$ by the second case of (4.1) in Theorem 4.4.

(3) If $p_{1}=3$ and $p_{2} \equiv 1(\bmod 9)$, or $p_{1} \equiv 1(\bmod 9)$ and $p_{2} \equiv 4$ or 7 $(\bmod 9)$, then $t=t_{K}=2$ by $(3.1)$ and $(3.2)$. By Theorem 4.4, 3-rank $K_{2} \mathcal{O}_{F}$ $=2-s_{K}+r$. Since $s_{K} \leq t_{K}=2$, we have 3-rank $K_{2} \mathcal{O}_{F}=0$ if and only if $s_{K}=2$ and $r=0$. We will prove that this is impossible. If $s_{K}=0$, then by Corollary 3.2 of [3], the class group $S_{K} \simeq \mathbb{Z} / 3 \mathbb{Z} \oplus \mathbb{Z} / 3 \mathbb{Z}$. Since the 3-rank of $S_{F}$ is also $2, S_{F} \simeq \mathbb{Z} / 3 \mathbb{Z}$. However if $r=0$, then $S_{F}^{1-\tau} \simeq \mathbb{Z} / 3 \mathbb{Z}$, which contradicts $s_{K}=0$. Hence 3 -rank $K_{2} \mathcal{O}_{F}>0$. Since $r \leq t-1=1$, the inequality 3 -rank $K_{2} \mathcal{O}_{F} \leq 3$ is obvious. 
Acknowledgements. The author is deeply grateful to the referee for helpful comments. He is also grateful to the Morningside Center of Mathematics in Beijing for hospitality.

\section{References}

[1] J. Browkin, Tame kernels of cubic cyclic fields, Math. Comp. 74 (2005), 967-999.

[2] A. Fröhlich, Central Extensions, Galois Groups, and Ideal Class Groups of Number Fields, Contemp. Math. 24, Amer. Math. Soc., Providence, RI, 1983.

[3] F. Gerth, On 3-class groups of cyclic cubic extensions of certain number fields, J. Number Theory 8 (1976), 84-98.

[4] -, On 3-class groups of pure cubic fields, J. Reine Angew. Math. 278/279 (1975), $52-62$.

[5] X. J. Guo, A remark on $K_{2}$ of the rings of integers of totally real number fields, Comm. Algebra, to appear.

[6] J. Hurrelbrink, $K_{2}(\mathcal{O})$ for two totally real fields of degree three and four, in: Algebraic $K$-theory, Part I (Oberwolfach, 1980), Lecture Notes in Math. 966, Springer, Berlin, $1982,112-114$.

[7] M. Ishida, The Genus Fields of Algebraic Number Fields, Lecture Notes in Math. 555, Springer, Berlin, 1976.

[8] F. Keune, On the structure of the $K_{2}$ of the ring of integers in a number field, $K$-Theory 2 (1989), 625-645.

[9] F. Kirchheimer, Über explizite Präsentationen Hilbertscher Modulgruppen zu totalreellen Körpern der Klassenzahl eins, J. Reine Angew. Math. 321 (1981), 120-137.

[10] D. Li, Defining equations and trace forms of cubic cyclic fields, Sci. China Ser. A 32 (1989), no. 1, 52-61.

[11] - Sextic cyclic fields containing the cube roots of unity, Kexue Tongbao 34 (1989), no. 2, 84-85 (in Chinese).

[12] - Class groups of cubic cyclic fields, Sichuan Daxue Xuebao 26 (1989), Special Issue, 132-135 (in Chinese).

[13] H. Y. Zhou, Tame kernels of cubic cyclic fields, Acta Arith. 124 (2006), 293-313.

Department of Mathematics

Nanjing University

Nanjing 210093, P.R. China

E-mail: guoxj@nju.edu.cn

Received on 29.3.2007

and in revised form on 18.7 .2007 